\title{
Synergistic gastric cancer inhibition by chemogenetherapy with recombinant human adenovirus p53 and epirubicin: An in vitro and in vivo study
}

\author{
YI-SHAN XIE $^{1 *}$, YAN-HUA ZHANG ${ }^{2,3 *}$, SHAO-PING LIU ${ }^{2}$, SHI-QUAN LIU ${ }^{2}$, \\ CHUN-WEI PENG ${ }^{2}$, LONG WU², HE-SHEN LUO ${ }^{1}$ and YAN LI ${ }^{2}$ \\ ${ }^{1}$ Department of Oncology, Renmin Hospital of Wuhan University, No. 238 Jiefang Road, Wuchang District, Wuhan 430060; \\ ${ }^{2}$ Departmen of Oncology, Zhongnan Hospital of Wuhan University and Hubei Key Laboratory of Tumor Biological \\ Behaviors, No. 169 Donghu Road, Wuchang District, Wuhan 430071, P.R. China
}

Received May 25, 2010; Accepted June 22, 2010

DOI: 10.3892/or_00001025

\begin{abstract}
This study was desinged to investigate the in vitro and in vivo antitumor effect on SGC-7901 gastric cancer cells by chemogenetherapy. SGC-7901 cells were treated by chemogenetherapy with Gendicine, a recombinant human Ad-p53 injection (rAd-p53), and epirubicin hydrochloride (EPI), a cytotoxic chemotherapy agent. Compared with blank control, rAd-p53, EPI, and combined therapy achieved SGC-7901 growth inhibition by $32.26,35.48,43.44 \%$, respectively on day 1 and $70.62,78.82,87.15 \%$, respectively on day 2 (rAdp53, EPI VS control, $\mathrm{p}<0.01$; rAd-p53+EPI VS either alone, $\mathrm{p}<0.05)$. Flow cytometry study confirmed that rAd-p53 and/or EPI mainly inhibit the cell cycle at $\mathrm{S}$ phase. SGC7901 cells were subcutaneously injected into the nude mice to form xenograft models, which were treated with rAd-p53 and EPI. Compared with the blank control, treatment with rAd-p53 at the dose of $10 \mu 1$ of $10^{12} \mathrm{vp} / \mathrm{ml}$ and EPI at the dose of $1.25 \mathrm{mg} / \mathrm{kg}, 7$ times in 3 weeks, resulted in 80 and $60 \%$ of tumor growth inhibition, respectively. No animal death was observed, although 2 nude mice in rAd-p53 group developed liver toxicity and 1 nude mouse in EPI
\end{abstract}

Correspondence to: Professor Yan Li, Departmen of Oncology, Zhongnan Hospital of Wuhan University, No. 169 Donghu Road, Wuchang District, Wuhan 430071, P.R. China E-mail: liyansd2@163.com

Professor He-Sheng Luo, Department of Gastroenterology, Remin Hospital of Wuhan University, No. 238, Jiefang Road, Wuchang District, Wuhan 430060, P.R. China

E-mail: luotang@public.wh.hb.cn

Present address: ${ }^{3}$ Department of Radiation Oncology, The First Affiliated Hospital of Kunming Medical College, No. 295 Xichang Road, Kunming 650032, P.R. China

*Contributed equally

Key words: gastric cancer, chemogenetherapy, recombinant human Ad-p53, epirubicin hydrocholoride, animal model group developed cardiac toxicity. rAd-p53 and EPI have synergistic tumor inhibition effect on gastric cancer cells.

\section{Introduction}

Gastric cancer (GC) is one of the most common malignant tumors in the world, and China alone accounts for $42 \%$ of all gastric cancers in the world (1), and a survey in 20042005 showed that GC is the third leading cause of cancer mortality in China (2). The disease in early stages is treatable by radical resection. However, $15-50 \%$ or more patients have developed a peritoneal carcinomatosis at the surgical exploration, thus curative treatment is no longer available $(3,4)$. To make the matter worse, it has long been recognized that GC does not generally respond to conventional chemotherapy (5-7). With the development of cancer genetics, gene therapy has stood out as a promising multidisciplinary treatment approach against GC.

The tumor suppressor p53 gene, located on chromosome $17 \mathrm{p} 13$, is the most frequently mutated gene in human cancers. Normal p53 gene (wild-type) regulates the cell cycle, DNA reparation, and cell apoptosis (8). Mutated p53 gene defect these functions and appeared to be carcinogenic (9). It is reported that $60 \%$ of human GC had a p53 gene mutation (10).

Gendicine (recombinant human Ad-p53 injection, rAdp53, Shenzhen SiBiono GeneTech Co., Ltd., Shenzhen, China) was the first commercial gene therapy product in the world, approved by the China State Food and Drug Administration in 2003, first for the treatment of head and neck cancers and later for other malignancies (11-14). It was based on a modified serotype 5 adenovirus vector engineered to express p53 gene to initiate the apoptosis pathway in the cell nucleus and the cytoplasm $(15,16)$. Gendicine can also promote drug sensitivity through a by-stander effect. It is reported that head and neck squamous cell carcinoma (HNSCC), lung cancer, breast cancer, and liver cancers have been treated with Gendicine and appeared to be effective and well tolerated $(15,16)$. Although the initial results were encouraging, it has also been recognized that gene therapy alone has not met the high expectations, and combination of gene therapy with other therapeutic approaches such 


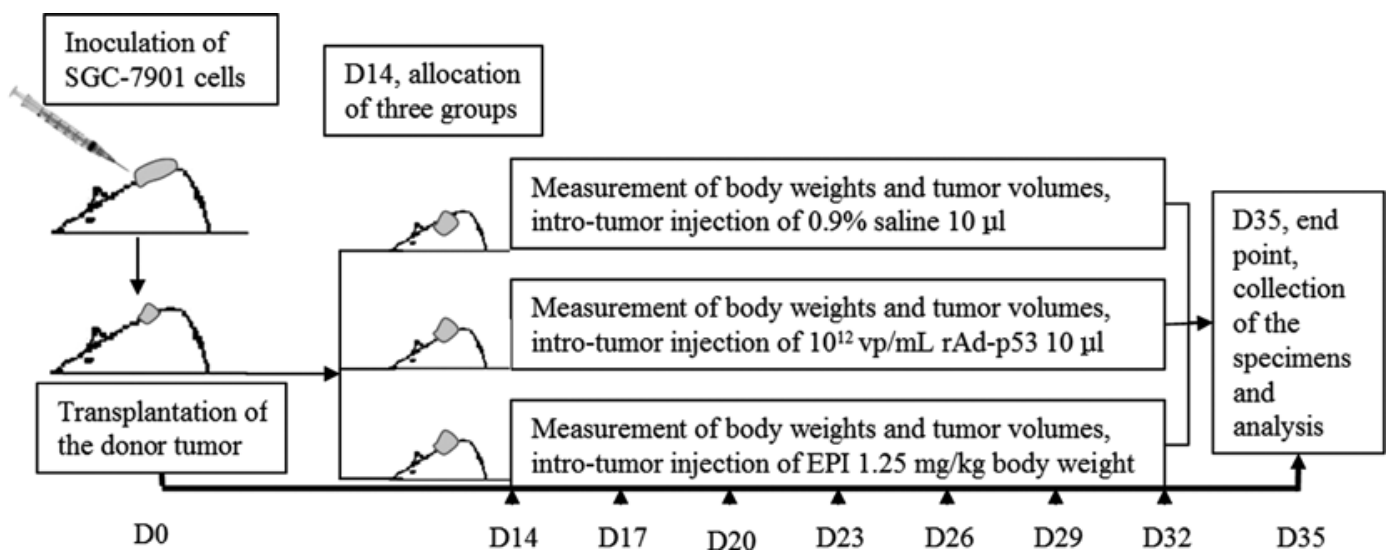

Figure 1. The in vivo study protocol and treatment schedule.

as chemotherapy might provide more treatment benefit. Therefore, this study was designed to explore the potential of recombinant human Ad-p53 in gastric caner treatment, either alone or in combination with chemotherapy, by in vitro and in vivo test.

\section{Materials and methods}

Agents. Recombinant Human Ad-p53 Injection (rAd-p53) (Gendicine, Shenzhen SiBiono GeneTech Co., Ltd., Shenzhen, China) $10^{12}$ viable particles (VP) per vial and epirubicin hydrochiloride for injection (EPI, Zhejiang Hisun Pharmaceuticals Co., Ltd., Zhejiang, China) $10 \mathrm{mg} /$ vial were obtained commercially. Drug storage and reconstitution were conducted according to the instructions of the manufacturer. RPMI-1640 medium (HyClone, NZ, USA) and Standard Newborn Bovine Serum (Zhengzhou Ben BioTech Co., Ltd., Zhengzhou, China) were obtained commercially. The biochemical kits for serum detection were obtained from Abbott Laboratories (Abbott Lab, IL, USA).

Cell lines and animals. Human gastric adenocarcinoma cell line SGC-7901 was provided by Hubei Key Laboratory of Tumor Biological Behaviors. Cells were cultured in RPMI-1640 medium supplemented with $10 \%$ standard newborn bovine serum in the $5 \% \mathrm{CO}_{2}$, saturated humidity, $37^{\circ} \mathrm{C}$ incubator (Shel Lab, OR, USA). The culture medium was changed every 2 days.

Female ALB/c-nu nude mice, 4-5 weeks old were obtained from Experimental Animal Center of HuBei Province (animal quality certificate, No. 00004509) and maintained in Animal Biosatety Level 3 Laboratory at the Experimental Center of Wuhan University. The animal study protocol was approved by the Animal Welfare Committee of the center.

Cell growth assay. SGC-7901 cells were plated in 24well culture plates (Corning, NY, USA), at a density of $3.6 \times 10^{4} /$ well. Four groups were treated with $10^{9} \mathrm{vp}$ of $\mathrm{rAd}-$ $\mathrm{p} 53,1 \mu \mathrm{g}$ of EPI, both of the two at the same dose, and a blank control with culture medium, respectively. To each well was added $1 \mathrm{ml}$ of culture medium. Cells of each group were harvested and counted daily with counting plate from day 1 (after $24 \mathrm{~h}$ ) to day 6 . The cell growth curve was drawn for each treatment group.
Flow cytometric analysis. The effect of the rAd-p53 and EPI on cell cycle progression was assessed by flow cytometry. SGC-7901 cells were plated in 6-well culture plates at a density of $5.0 \times 10^{5} /$ well, treated with $5 \times 10^{9}$ vp of rAd-p53, $1 \mu \mathrm{g}$ EPI, both of the two, and a blank control with culture medium. Each well was added with $3 \mathrm{ml}$ medium. Cells were collected after $48 \mathrm{~h}$ incubation, digested with $0.25 \%$ trypsin/ EDTA to make single-cell suspension, $4^{\circ} \mathrm{C}$ x $1000 \mathrm{rpm}$ centrifugation for $10 \mathrm{~min}$, washed twice with $\mathrm{PBS}$, fixed and stained with PI agents kit (Beckman Coulter, CA, USA) following the instructions. The fluorescence of cells was measured with FC 500 (Beckman Coulter).

Cytomorphology study. SGC-7901 cells were plated in 6-well culture plates with cover slips for $48 \mathrm{~h}$ as above. When cell confluence reached $80 \%$, the cover slips were removed, washed twice with PBS, fixed in glacial methanol for $30 \mathrm{~min}$. The slips were stained with Wright's stain for $15 \mathrm{~min}$ at room temperature after being airdried. Cover slips were washed completely and cellular morphology was observed under a microscope.

Tumor xenograft models and therapy schedule. SGC-7901 cells $\left(5 \times 10^{6}\right)$ were subcutaneously inoculated into the back of one nude mouse as a donor tumor, which was resected when it reaches $8-10 \mathrm{~mm}$ in diameter after 20 days and cut into cubes $\sim 2 \mathrm{~mm}$ in diameter, then transplanted under the left dorsal skin of 4-5-week old female nude mice surgically under sterile conditions. Tumor length and width were measured with a sliding caliper a week after inoculation. Tumor volume was estimated from length $\mathrm{x}$ width $2 / 2$ and presented as mean (SD) $\mathrm{mm}^{3}$. When the tumors reached 3-5 $\mathrm{mm}$ in diameter, the tumor-bearing nude mice were allocated to three groups, each with approximately equal means and standard errors of the estimated tumor volume. The rAd-p53 therapy group ( $n=8)$, EPI therapy group $(n=8)$, and the control group $(\mathrm{n}=6)$ were given $10 \mu \mathrm{l}$ of $10^{12} \mathrm{vp} / \mathrm{ml}$ of rAd-p53, $1.25 \mathrm{mg} / \mathrm{kg}$ body weight of EPI $(10 \mu \mathrm{l})$, and $10 \mu \mathrm{l}$ of $0.9 \%$ saline, respectively. Each mouse received intratumor injection of the study drugs every three days over a period of three weeks, from a different direction each time. The body weights and tumor volumes were measured before every injection. Two days after the last injection, all nude mice were euthanized. The blood, tumor, major organs such as 


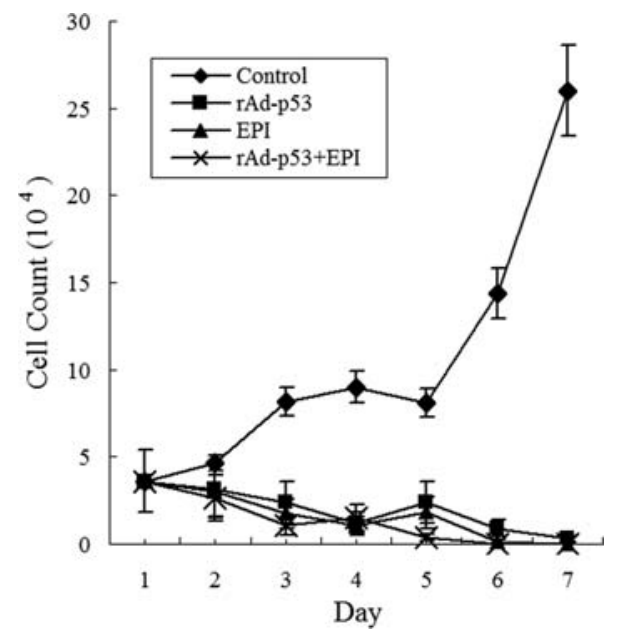

Figure 2. The inhibition of rAd-p53 and/or EPI on SGC-7901 cells. Both rAd-p53 and EPI significantly inhibit the growth of SGC-7901 cells, and the combination of the two drugs achieves greater inhibition, suggesting synergism between rAd-p53 and EPI.

the heart, the kidneys, the lungs and the liver were collected for biochemical and histological studies. The detail study protocol is depicted in Fig. 1.

Histological study. Formalin-fixed, paraffin-embedded tumor tissues were cut at $5-\mu \mathrm{m}$ thickness. Tumor feature were observed under light microscope after henatoxylin and eosin (H\&E) staining.

Western blot assay. Tumors of each group were lysed and protein extracted with ProteoExtract ${ }^{\circledR}$ subcellular proteome extraction kit (Meck, Germany) according to the protocol. Protein concentration of each sample was determined after the BCA assay using a protein assay kit from Pierce (Rockford, IL, USA). Equal amounts of protein were loaded onto $12 \%$ gels (Invitrogen, CA, USA) (50 $\mu \mathrm{g} /$ lane) and separated by SDS-PAGE, electrophoretically transferred to PVDF membranes (Immobilon $0.2 \mu \mathrm{m}$ ) (Millipore, CA, USA). After blocking with 5\% skimmed milk in washing buffer containing $140 \mathrm{mM} \mathrm{NaCl}, 25 \mathrm{mM}$ Tris $\mathrm{HCl} \mathrm{pH} \mathrm{7.8,}$ and $0.05 \%$ Tween-20 for $2 \mathrm{~h}$ at room temperature, membranes were washed and incubated with rabbit anti-p53 (Santa Cruz, CA, USA) at 1:200 or rabbit anti-actin (Santa Cruz) at $1: 1000$ overnight at $4^{\circ} \mathrm{C}$ and then with the corresponding peroxidase linked secondary antibodies at room temperature for $1 \mathrm{~h}$ at 1:2000. Enhanced chemiluminescence (ECL, Amersham Biosciences, NJ, USA) was used to visualize the immunoreactive bands. All bands were scanned and analyzed by Syngene GeneGenius bioimaging systems (Synoptics Ltd., UK). The gray scale ratio of each treatment group was calculated as gray scale of target protein/gray scale of actin.

Toxicity study. The serum of each nude mouse was diluted at a 1:10 ratio with $0.9 \%$ saline. The liver, renal and heart functions were studied in serum of each nude mouse using Abbott Biochemical Analyzer (Abbott Lab). Histopathological study of the liver and heart were also performed on paraffin-embedded specimens with H\&E staining.

Statistical analysis. The data were analyzed on SPSS software version 13.0 (SPSS Inc., Chicago, IL, USA). The differences in the inhibitions and cell cycles among different therapy groups were compared using t-test. The differences in tumor sizes among different therapy groups were compared using Mann-Whitney U-test at each time point. Body weights of different groups were compared using ANOVA at each time point. $\mathrm{p}<0.05$ was considered to be statistically significant.

\section{Results}

Inhibition of GC cell growth. As shown in Fig. 2, the inhibition rate of rAd-p53, EPI, combined therapy on SGC7901 were $32.26,35.48,43.44 \%$ on day 1 and $70.62,78.82$, $87.15 \%$ on day 2, respectively. rAd-p53 and EPI inhibited the growth of SGC-7901 cells effectively compared to the blank control $(\mathrm{p}<0.01)$, and rAd-p53 combined with EPI clearly improved the inhibition $(\mathrm{p}<0.05)$. After treating SGC7901 cells for 5 days, the inhibition rate reached $93.75 \%$ for rAd-p53 and $98.96 \%$ for EPI. The combination of them resulted in $\sim 100 \%$ inhibition of growth $(\mathrm{p}<0.01)$ (Fig. 2).

Effect of the rAd-p53 on the cell cycle and apoptosis. The effect of rAd-p53 and/or EPI on the cell cycle is shown in Fig. 3. As compared to the controlled group, rAd-p53 and/ or EPI affected the SGC-7901 cell cycle. The percentage of cells in phase G0/G1 was decreased and phase $S$ was increased compared to the control $(\mathrm{p}<0.05)$ (Table I). An obvious sub-G1 peak in the rAd-p53 treated group and the combined group were observed in Fig. 3, indicating apoptotic cells.

Table I. Cell cycle and apoptosis of different treatment groups.

\begin{tabular}{lcrr}
\hline Groups & G0/G1 $(\%)$ & S $(\%)$ & G2/M $(\%)$ \\
\hline Control & $46.357 \pm 5.835^{\mathrm{a}}$ & $29.945 \pm 6.742^{\mathrm{a}}$ & $23.700 \pm 5.903$ \\
rAd-p53 & $28.828 \pm 6.143$ & $52.699 \pm 9.152$ & $18.473 \pm 6.191$ \\
EPI & $4.831 \pm 3.746$ & $91.144 \pm 5.981$ & $4.023 \pm 2.564$ \\
rAd-p53+EPI & $15.073 \pm 11.094$ & $65.858 \pm 13.423$ & $19.068 \pm 9.562$ \\
\hline
\end{tabular}

${ }^{\mathrm{a}} \mathrm{p}<0.05$ compared with treatment groups. 

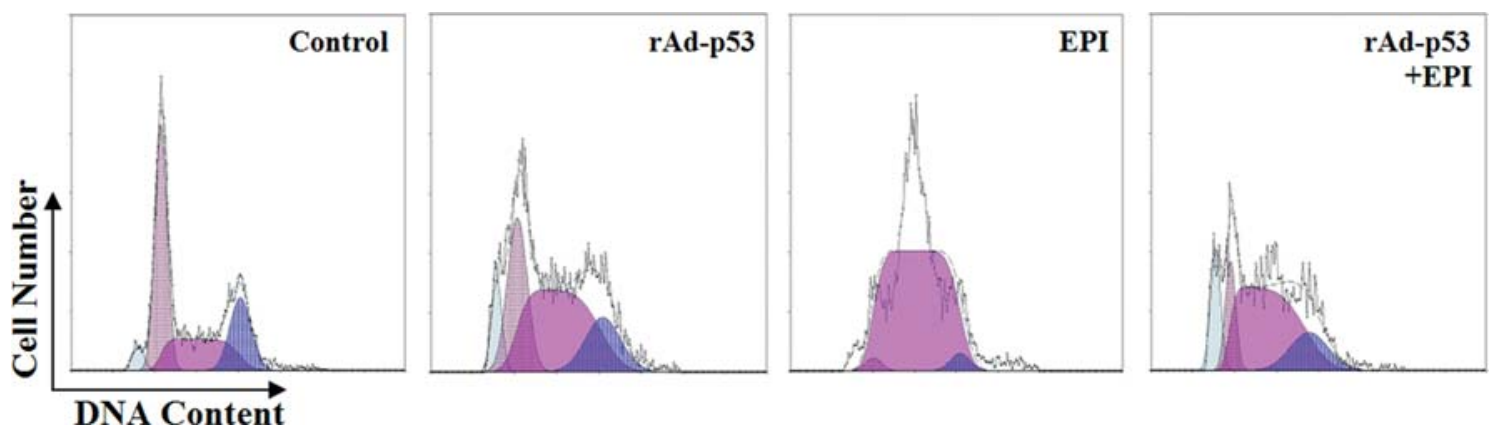

Figure 3. Analysis of cell cycle by flow cytometry. Both rAd-p53 and EPI mainly block the cell cycle at phase S.

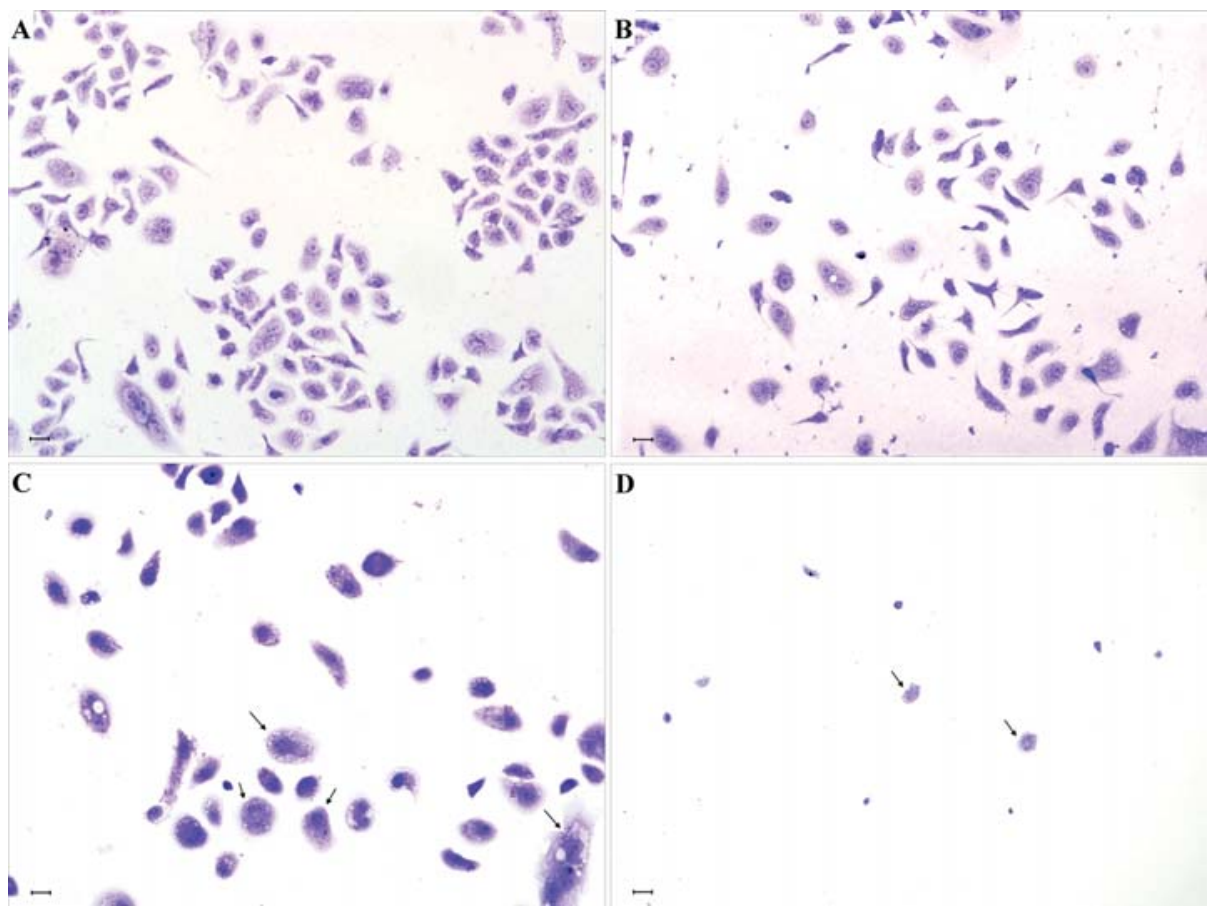

Figure 4. Treatment effects on SGC-7901 morphology. Compared with blank control (A), EPI treatment at $1 \mu \mathrm{g}$ for 2 days results in obvious cell shrinkage, decreased cell number and increased cell death debris (B), rAd-p53 treatment at $5 \times 10^{9}$ vp for 2 days results in increased cell apoptosis (C), and the combination of rAd-p53 and EPI treatment results in total cell death (D); magnification, x200; Wright's staining; scale bar, $20 \mu \mathrm{m}$. Arrow marked: apoptotic cells.

Cytomorphology and pathology. After Wright staining the control SGC-7901 cells were fusiform, grown to confluence, the cell structures were complete and clear (Fig. 4A). The confluent cells in EPI treated group seemed less than the control, and dispersed cells appeared (Fig. 4B). In Fig. 4C, the junction between cells could not been seen after treating with rAd-p53 for $48 \mathrm{~h}$. The number of SGC-7901 cells were obviously decreased, the cells were dispersed, single, changed from normal shape. Cytoplasm was stained deeper and nucleolus was disrupted into small pieces, several apoptotic cells were seen under the microscope (Fig. 4C). Cells were rare in the combined treatment group, several cell fragments could be seen(Fig. 4D), and there were few complete cells.

Inhibition of tumor growth in nude mice. We implanted the SGC-7901 cells into nude mice and assessed the efficacy of rAd-p53 in vivo. On day 14 after the implantation, tumors on the left dorsal skin of all nude mice grew to $3-5 \mathrm{~mm}$ in

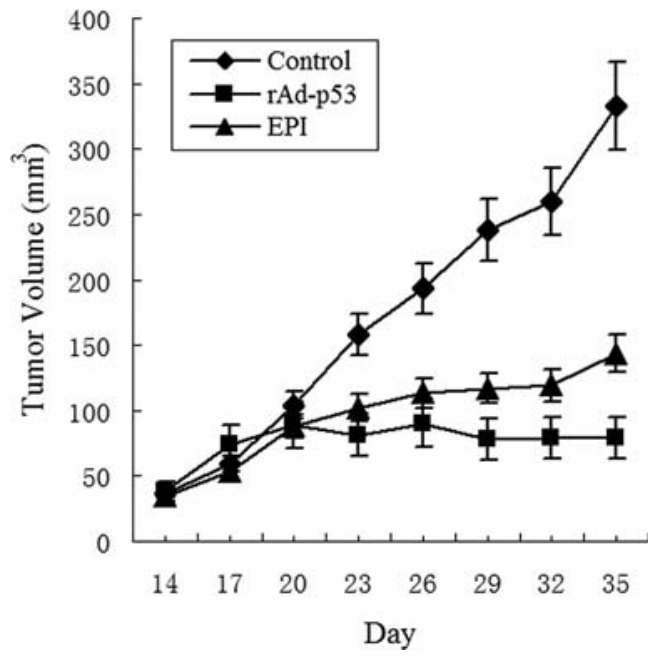

Figure 5. Tumor growth of different therapy groups. Both rAd-p53 and EPI significantly inhibit tumor growth and rAd-p53 has sustained and similar tumor inhibition effect to EPI. 

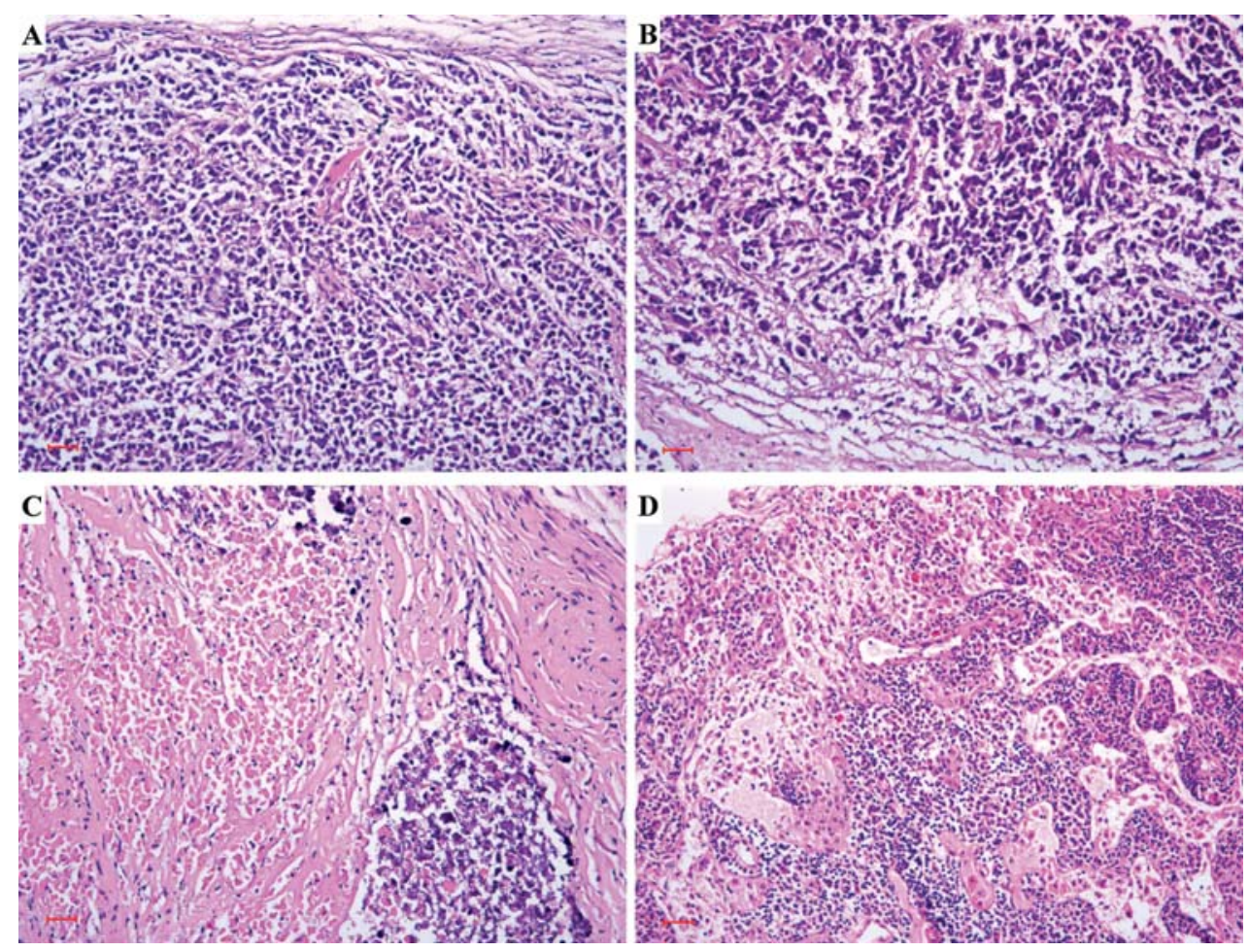

Figure 6. Micrographs of subcutaneous tumors under different treatment approaches. (A) Sodium chloride solution (10 $\mu 1$; $0.9 \%$ ) 7 injections, no tumor necrosis was observed. (B) EPI $(1.25 \mathrm{mg} / \mathrm{kg}) \times 7$ injections, decreased tumor cell density and increased fibrosis were observed. (C) rAd-p53 (10 $\mu 1)$ at the dose of $10^{12} \mathrm{vp} / \mathrm{ml} \mathrm{x} 7$ injections, conspicuous tumor necrosis was observed. (D) Therapy was the same as (C), conspicuous lymphocyte infiltration was evident. Hematoxylin and eosin stain; magnification, x200. Scale bar, $20 \mu \mathrm{m}$.

diameter. The three different therapies were performed under the schedule shown in Fig. 1. Fig. 5 shows the tumor growth of the three groups, the tumor volume of rAd-p53 group was significantly smaller than the control group from the sixth injection to the end point $(\mathrm{p}<0.01)$. The difference in rAd-p53 and EPI therapy group was not statistically significant. The weight of the three groups over the period of therapy was not different $(\mathrm{p}>0.05)$.

Pathology and the expression of p53 protein. The $\mathrm{H} \& \mathrm{E}$ stained tumor slices were observed under light microscope. In the control group, there was no necrosis found, and tumor shape and structure were well stained (Fig. 6A). Necrosis in the tumor tissue of nude mice in the EPI treated group was not obvious (Fig. 6B). Remarkable necrosis was observed in the tumor tissue of nude mice treated with rAd-p53 (Fig. 6C), and there was some leukomonocytes infiltrating the tumor (Fig. 6D).

Western blotting of the $\mathrm{p} 53$ protein among different groups is shown in Fig. 7. The gray scale ratios of rAd-p53, EPI and control groups were $0.234,1.012$ and 1.309 , respectively.

Side reaction. The serum biochemical parameters were tested to analyze the toxicity of the therapy agents (Table II). The alanine aminotransferase (ALT), aspartate aminotransferase (AST), lactate dehydrogenase (LDH) in control group was $92.5 \pm 8.86 \mathrm{U} / 1,198.75 \pm 50.83 \mathrm{U} / 1,1227.5 \pm 314.22 \mathrm{U} / 1$, respectively. Two nude mice in the rAd-p53 group had a remarkable increase of the above three parameters (Table II).

\section{EPI Control rAd-p53}

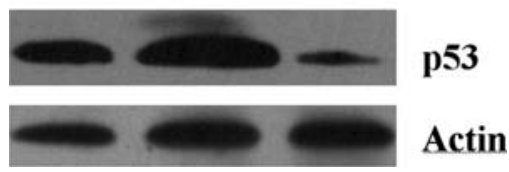

Figure 7. Western blotting of the treatment groups

The results were validated by liver H\&E staining, which showed the two livers had serious hepatitis (Fig. 8). The creatine kinase $(\mathrm{CK})$ and myoglobin isoenzyme of creatine kinase (CK-MB) in control group were $743.75 \pm 214.339 \mathrm{U} / \mathrm{l}$, $773.75 \pm 227.718 \mathrm{U} / 1$, respectively. One case in the EPI group had a remarkable increase of the two enzymes, and the result was validated by histopathology (Fig. 9). The index of renal function (BUN) of the EPI treatment group was significantly higher than the other two groups $(\mathrm{p}<0.05)$. Other enzymes in nude mice in therapy groups did not show any abnormal signs.

\section{Discussion}

Due to an extremely close relationship to human malignant tumor (17), the p53 gene has been one of the most promising targets for cancer gene therapy. The high resistance to anticancer drug is an important cause of therapy failure in GC. The p53 gene therapy for GC was proved effective in the study of Ohashi et al (18). The recombinant human Ad-p53 has been previously confirmed to be effective against head 
Table II. Detailed information on key parameters of nude mice liver, renal and cardiact functions.

\begin{tabular}{|c|c|c|c|c|c|c|c|}
\hline Nude mice & $\operatorname{ALT}(10 \mathrm{U} / 1)$ & AST (10 U/l) & $\mathrm{LDH}(\mathrm{U} / \mathrm{l})$ & CK (10 U/1) & CK-MB (10 U/1) & BUN (10 mmol/l) & $\mathrm{Cr}(10 \mu \mathrm{mol} / \mathrm{l})$ \\
\hline \multicolumn{8}{|l|}{ Control } \\
\hline 1 & 9 & 19 & 108 & 84 & 85 & 1.06 & 17.4 \\
\hline 2 & 10 & 21 & 160 & 85 & 89 & 0.69 & 19.4 \\
\hline 3 & 8 & 18 & 142 & 82 & 87 & 1.16 & 20.4 \\
\hline 4 & 10 & 31 & 145 & 70 & 75 & 0.89 & 19.4 \\
\hline 5 & 8 & 13 & 57 & 58 & 56 & 0.31 & 20.1 \\
\hline 6 & 10 & 18 & 115 & 54 & 54 & 0.64 & 18.7 \\
\hline \multicolumn{8}{|l|}{ rAd-p53 } \\
\hline 1 & 8 & 17 & 144 & 82 & 88 & 0.6 & 19.4 \\
\hline 2 & 209 & 226 & 452 & 43 & 51 & 0.7 & 21.1 \\
\hline 3 & 11 & 18 & 127 & 77 & 82 & 0.96 & 18.4 \\
\hline 4 & 647 & 690 & 1189 & 84 & 89 & 0.56 & 19.1 \\
\hline 5 & 12 & 23 & 209 & 43 & 50 & 0.76 & 20.4 \\
\hline 6 & 11 & 20 & 130 & 54 & 61 & 0.68 & 19.6 \\
\hline 7 & 9 & 17 & 145 & 76 & 82 & 0.82 & 20.1 \\
\hline 8 & 10 & 19 & 180 & 48 & 53 & 0.7 & 18.7 \\
\hline \multicolumn{8}{|l|}{$\mathrm{EPI}^{\mathrm{a}}$} \\
\hline 1 & 8 & 16 & 127 & 53 & 57 & 1.1 & 15.3 \\
\hline 2 & 27 & 24 & 115 & 85 & 84 & 1.2 & 14.9 \\
\hline 3 & 12 & 21 & 211 & 171 & 171 & 1.1 & 14.5 \\
\hline 4 & 12 & 17 & 103 & 59 & 62 & 1.2 & 15 \\
\hline 5 & 10 & 21 & 170 & 76 & 77 & 1.3 & 15.1 \\
\hline 6 & 14 & 20 & 145 & 56 & 58 & 1.2 & 14.8 \\
\hline 7 & 10 & 22 & 160 & 53 & 59 & 1.1 & 15.2 \\
\hline 8 & 9 & 18 & 205 & 75 & 82 & 0.89 & 15.1 \\
\hline
\end{tabular}

${ }^{\mathrm{a}} \mathrm{p}<0.05$, EPI group had higher BUN level than the rAd-53 group or the blank control. ALT, alanine aminotransferase; AST, aspartate aminotransferase; LDH, lactate dehydrogenase; CK, creatine kinase; CK-MB, myoglobin isoenzyme of creatine kinase; BUN, blood urea nitrogen; $\mathrm{Cr}$, creatinine. Black bold figures: abnormal values.

and neck cancers, with clinically acceptable adverse events (11-14). The present study explored the treatment potential of this novel agent against GC by in vitro and in vivo experiments.

After being treated by rAd-p53 and/or EPI for just $24 \mathrm{~h}$, SGC-7901 cells showed a significant growth inhibition compared with blank control $(\mathrm{p}<0.01)$. The combined rAdp53 and chemotherapy agent EPI showed a synergetic effect, which reached $87.15 \%$ of cell growth inhibition after 2 days $(\mathrm{p}<0.05)$, and $\sim 100 \%$ after 5 days. Flow cytometric analysis demonstrated that rAd-p53 mainly blocked the cell cycle at phase $\mathrm{S}$. Moreover, the increased sub-G1 peak also suggested enhancement of apoptotic effect of rAdp53 ( $<<0.05)$. We also studied the morphology of SGC-7901 cells by different treatments after Wright's staining. The cell numbers on the cover slips of rAd-p53 and/or EPI treatment group were decreased remarkably, especially for the combined therapy group. The shape of EPI treated cells did not change, and apoptotic cells were rare. However, the cells treated with rAd-p53 seemed larger than in other groups, and apoptotic cells were frequently seen in the slips. rAd-p53 combined with EPI had a synergetic effect.
EPI is an anti-cancer drug of anthracene nucleus category, which inhibits the DNA/RNA synthesis and mitosis by directly inserting into the DNA base pairs to generate a compound which interrupt mRNA transcription. The effect of EPI depends on the apoptosis pathway (19).

The wild-type p53 gene exerts its cytoprotective effect by the $\mathrm{p} 53$ protein expression. In normal cells, such p53 protein expression is low because no cell damage signals are present. However, when DNA damage has occurred p53 protein degradation will be significantly reduced leading to high level accumulation of p53 protein, which in turn triggers apoptosis (17). Therefore, when EPI and rAd-p53 were used in combination, the direct DNA damage effect of EPI could activate the wt p53 gene, resulting in enhanced functional rAd-p53 and increased apoptosis $(14,20)$. Such increased apoptosis will in turn potentiate the cell damage effect of EPI. Therefore, EPI and p53 protein could form a positive feedback loop, in which the effect of these agents augments one another (Fig. 10).

The in vivo study confirmed that rAd-p53 had similar tumor inhibition effect to EPI ( $p>0.05)$, but significantly greater effect than the control group $(\mathrm{p}<0.01)$. Histological 

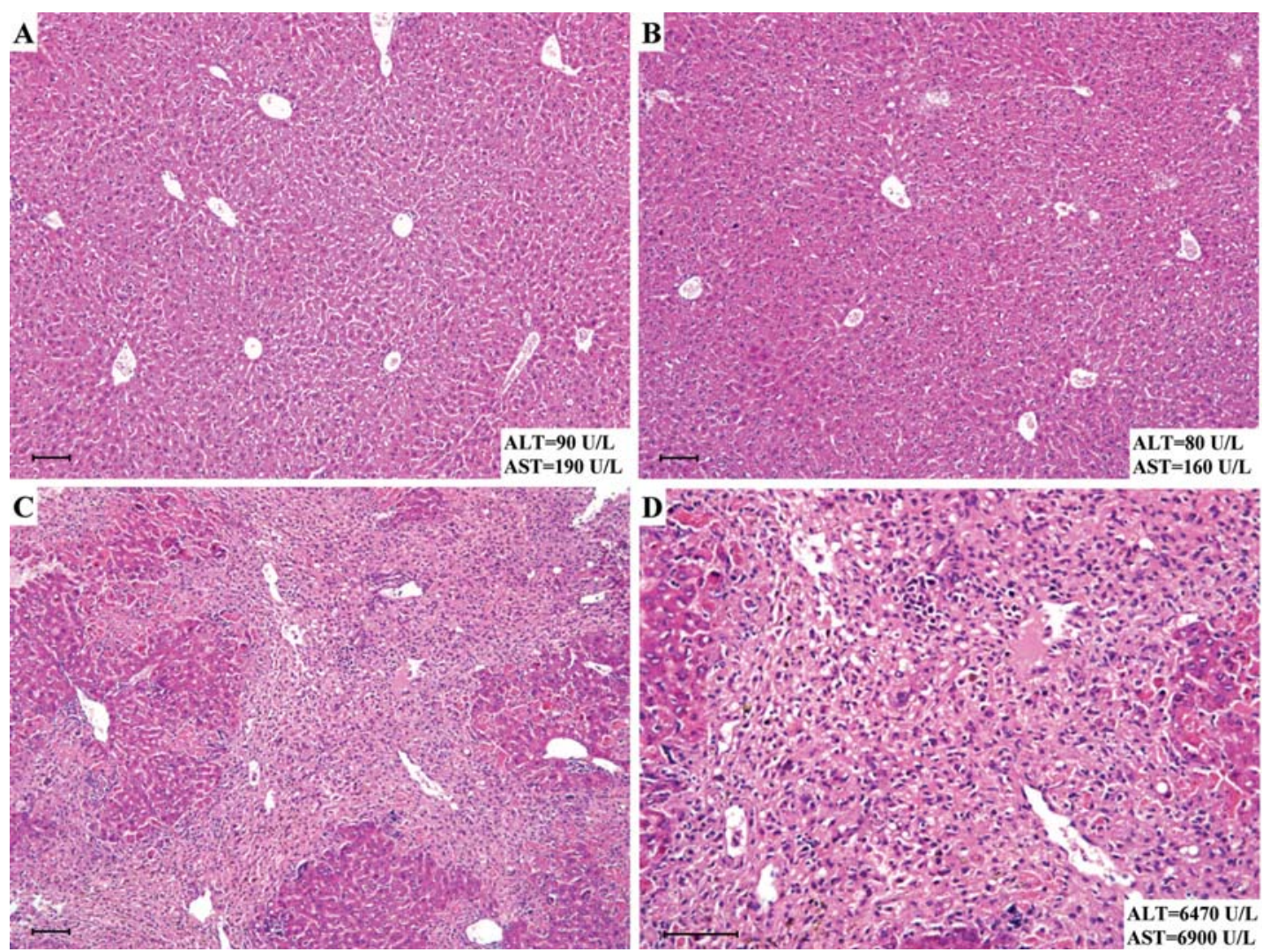

Figure 8. Histopathology of the liver of different treatment groups. (A) Sodium chloride solution $(10 \mu 1,0.9 \%) \times 7$ injections, normal liver structure was evident. (B) EPI $(1.25 \mathrm{mg} / \mathrm{kg}) \times 7$ injections, no inflammation was observed. (C) rAd-p53 (10 $\mu 1)$ at the dose of $10^{12} \mathrm{vp} / \mathrm{ml} \times \mathrm{x}$ injections, conspicuous liver inflammation and necrosis were observed in 2 of 8 nude mice (x100). (D) A magnification of (C) showing inflammatory cell infiltration (x200). Hematoxylin and eosin stain, scale bar, $50 \mu \mathrm{m}$; the inserts on the right lower corner in each graph show the liver enzyme levels.
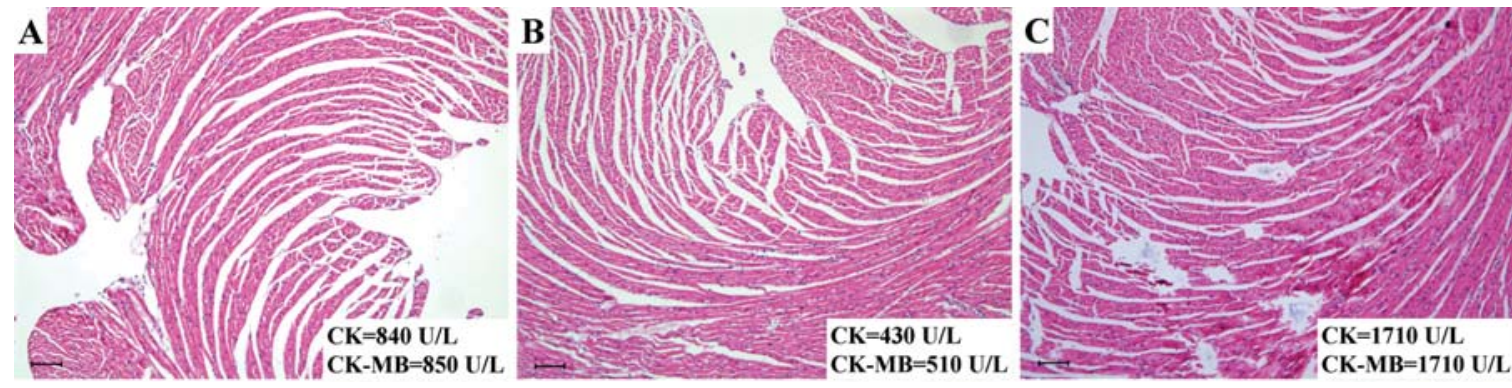

Figure 9. Histopathology of the myocardium of different treatment groups. (A) Sodium chloride solution (10 $\mu 1 ; 0.9 \%) \times 7$ injections, normal myocardium structure. (B) rAd-p53 (10 $\mu 1)$ at the dose of $10^{12} \mathrm{vp} / \mathrm{ml} \mathrm{x} 7$ injections, normal myocardium structure with no signs of damage. (C) EPI (1.25 mg/kg) x 7 injections, scattered piecemeal myocardium necrosis were observed in 1 of 8 nude mice. Magnification, x100; hematoxylin and eosin stain; scale bar, $50 \mu \mathrm{m}$; the inserts on the right lower corner in each graph show the heart enzyme levels.

study revealed conspicuous tumor necrosis and apoptosis in the rAd-p53 treatment group. We performed Western blotting to measure p53 protein expression and found p53 protein expression level was lower in rAd-p53 and EPI groups than control group. This could be explained by the fact that $\mathrm{p} 53$ protein expressed by the mutational type (mt) p53 gene has a longer half-life than that expressed by the wt gene (21-23), and gastric cancer SGC-7901 cells have p53 gene mutation (24). As the tumor specimens were harvested 3 days after the last injection, so the p53 protein detected in this study could be the mt. Therefore, the results of Western blotting suggested that rAd-p53 suppressed mt p53.

The rAd-p53 is generally safe and well tolerated in the clinical studies in human head and neck cancers (11-14). However, safety data on nude mice are inadequate. In this study, 2 of 8 nude mice in the rAd-p53 group developed liver toxicity, characterized by the significantly increased serum ALT, AST and LDH levels, diffused hepatocyte necrosis with lymphocyte infiltration. No special treatments were delivered to these animals. Nevertheless, there were no 


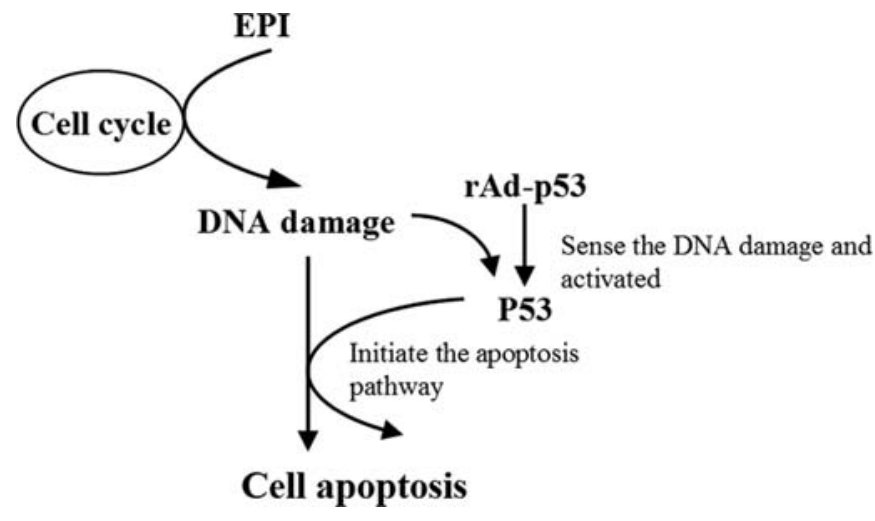

Figure 10. The mechanism of EPI combined with rAd-p53.

deaths in these animals during the study period. Such liver toxicity did not occur in the EPI group or the control group, suggesting this could be directly due to the adenovirus vector. This could also be attributed to the fact that nude mice are immunodeficiency animals without any cellular immunity, thus are extremely vulnerable to virus. In another study by Zhang et al (25), who evaluated a self-constructed adenovirus vector, similar liver toxic effects were also recorded. This information suggests that liver protection should be a key consideration in the use of rAd-p53.

This study also confirmed another well recognized cardiac toxicity of anthracene nucleus category drugs. In the EPI treatment group, 1 of 8 nude mice developed cardiac toxicity, characterized by the significantly increased serum $\mathrm{CK}$ and CK-MB levels, and scattered piecemeal myocardium necrosis. Again, no unexpected animal deaths occurred in this group during the study period, suggesting that the EPI used at the study dosage was safe.

In summary, this study demonstrated that rAd-p53 significantly inhibited gastric cancer SGC-7901 cells both in vitro and in vivo, and rAd-p53 had synergetic effect with EPI. This opens a new possibility of chemogenetherapy for gastric cancer.

\section{Acknowledgements}

This study was supported by New-Century Excellent Talents Supporting Program of the Ministry of Education of China (NCET-04-0669), Foundation for the Author of National Excellent Doctoral Dissertation of China (FANEDD-200464), and the Science Fund for Creative Research Groups of the National Natural Science Foundation of China (No. 20921062).

\section{References}

1. Parkin DM, Bray F, Ferlay J and Pisani P: Global cancer statistics, 2002. CA Cancer J Clin 55: 74-108, 2005.

2. Jemal A, Siegel R, Ward E, et al: Cancer Statistics, 2006. CA Cancer J Clin 56: 106-130, 2006.

3. Bozzetti F, Yu W, Baratti D, et al: Logoregional treatment of peritoneal carcinomatosis from gastric cancer. J Surg Oncol 98: 273-276, 2008.
4. Archie V, Kauh J, Jones DV Jr, et al: Gastric cancer: standards for the 21st century. Crit Rev Oncol Hematol 57: 123-131, 2006.

5. Leong T: Chemotherapy and radiotherapy in the management of gastric cancer. Curr Opin Gastroenterol 21: 673-678, 2005.

6. Dicken BJ, Bigam DL, Cass C, et al: Gastric adenocarcinoma: review and considerations for future directions. Ann Surg 241: 27-39, 2005.

7. Zhang D and Fan D: Multidrug resistance in gastric cancer: recent research advances and ongoing therapeutic challenges. Expert Rev Anticancer Ther 7: 1369-1378, 2007.

8. Bouchet BP, de Fromentel CC, Puisieux A and Galmarini CM: p53 as a target for anti-cancer drug development. Crit Rev Oncol Hematol 58: 190-207, 2006.

9. Nabeya Y, Loganzo F Jr, Maslak P, et al: The mutational status of p53 protein in gastric and esophageal adenocarcinoma cell lines predicts sensitivity to chemotherapeutic agents. Int J Cancer 64: 37-46, 1995.

10. Van Dillen IJ, Mulder NH, Vaalburg W, et al: Influence of the bystander effect on HSV-tk/GCV gene therapy. Curr Gene Ther 2: 307-322, 2002

11. Han DM, Huang ZH, Zhang W, et al: Effectiveness of recombinant adenovirus p53 infection on laryngeal cancer: phase I clinical trial and follow up. Natl Med J China 83: 2029-2032, 2003. (In Chinese with English abstract)

12. Chen CB, Pan JJ and Xu LY: Recombinant adenovirus p53 agent injection combince with radiotherapy in treatment of nasopharyngeal carcinoma: a phase II clinical trial. Nat Med J China 83: 2033-2035, 2003. (In Chinese with English abstract)

13. Swisher SG, Roth JA, Komaki R, et al: Induction of p53regulated genes and tumor regression in lung cancer patients after intratumoral delivery of adenoviral p53 (INGN 201) and radiation therapy. Clin Cancer Res 9: 93-101, 2003

14. Zhang S, Li Y, Li L, et al: Phase I study of repeated intraepithelial delivery of adenoviral p53 in patients with dysplastic oral leukoplakia. J Oral Maxillofac Surg 67: 1074-1082, 2009.

15. Wilson JM: Gendicine: the first commercial gene therapy product. Hum Gene Ther 16: 1014-1015, 2005.

16. Peng Z: Current status of gendicine in China: recombinant human Ad-p53 agent for treatment of cancers. Hum Gene Ther 16: 1016-1027, 2005.

17. Fridman JS and Lowe SW: Control of apoptosis by p53. Oncogene 22: 9030-9040, 2003.

18. Ohashi M, Kanai F, Ueno H, et al: Adenovirus mediated p53 tumour suppressor gene therapy for human gastric cancer cells in vitro and in vivo. Gut 44: 366-371, 1999.

19. Bunz F, Hwang PM, Torrance C, et al: Disruption of p53 in human cancer cells alters the responses to therapeutic agents. J Clin Invest 104: 263-269, 1999.

20. Schmitt CA and Lowe SW: Apoptosis is critical for drug response in vivo. Drug Resist Updat 4: 132-134, 2001.

21. Pinto-de-Sousa J, Silva F, David L, et al: Clinicopathological significance and survival influence of p53 protein expression in gastric carcinoma. Histopathology 44: 323-331, 2004.

22. Shiao YH, Palli D, Caporaso NE, et al: Genetic and immunohistochemical analyses of p53 independently predict regional metastasis of gastric cancers. Cancer Epidemiol Biomarkers Prev 9: 631-633, 2000

23. Fenoglio-Preiser CM, Wang J, Stemmermann GN and Noffsinger A: TP53 and gastric carcinoma: a review. Hum Mutat 21: 258-270, 2003.

24. Zhao AG, Zhao HL, Jin XJ, et al: Effects of Chinese Jianpi herbs on cell apoptosis and related gene expression in human gastric cancer grafted onto nude mice. World J Gastroenterol 8: 792-796, 2002.

25. Zhang WW, Alemany R, Wang J, et al: Safety evaluation of Ad5CMV-p53 in vitro and in vivo. Hum Gene Ther 6: 155-164, 1995. 